
\title{
CIÊNCIA'NATURA
}

\section{Observação direta da turbulência atmosférica na pluma emitida por uma Usina Termoelétrica}

\author{
Direct observation of the atmospheric turbulence in the plume emitted by a Thermoelectric \\ Plant
}
${ }^{1}$ Luís Gustavo Nogueira Martins, ${ }^{1}$ Otávio Costa Acevedo, ${ }^{1}$ Franciano Scremin Puhales, ${ }^{1}$ Pablo Eli Soares De Oliveira, ${ }^{1}$ Cláudio Alberto Teichrieb, ${ }^{1}$ Samuel Silva, ${ }^{1}$ Giuliano Demarco e
${ }^{1}$ Felipe Denardin Costa

${ }^{1}$ Universidade Federal de Santa Maria, Santa Maria, RS, Brasil

\section{Resumo}

Uma torre micrometeorológica com 11 níveis de observação de turbulência está em operação junto à Usina Termoelétrica da Linhares Geração SA, desde agosto de 2016. Em casos particulares, quando o vento sopra na direção da Usina para a torre, é possível observar diretamente as características físicas da pluma emitida, como turbulência e fluxos no interior da pluma de emissão. No presente trabalho, é analisado um caso em que isto ocorreu. A análise mostra que estes eventos são marcados por grande aumento das flutuações de velocidade do vento e de temperatura do ar, que são iniciados exatamente quando a direção do vento se torna de Sul, direção em que se situa a Usina em relação à torre. A seguir, se comprova que estes eventos de grandes flutuações são de fato sinais turbulentos, pois os espectros das séries temporais observadas no período seguem a Lei de Kolmogorov. Finalmente, são apresentadas as evoluções temporais dos perfis verticais de energia cinética turbulenta e fluxo turbulento de calor no período de passagem da pluma pela torre.

Palavras-chave: Camada Limite Atmosférica; Dispersão; Espectros Turbulentos, Turbulência Atmosférica.

\begin{abstract}
A micrometeorological tower with 11 levels of turbulence observation has been in operation near to the Linhares Geração SA Thermoelectric Plant since August 2016. In particular cases, when the wind blows towards the tower, it is possible to observe directly the physical characteristics of the emitted plume, such as the turbulence and fluxes inside the emitted plume. In the present work, a case is analyzed in which it has occurred. The analysis shows that these events are characterized by an increase in the air wind speed and temperature fluctuations, which starts exactly when the wind direction becomes south, direction which the power plant is located in relation to the tower. Next, it is proved that these events of increase of the fluctuations are in fact turbulent signs, the spectra of the observed time series during the period follow the Law of Kolmogorov. Finally, it is presented the temporal evolutions of the vertical profiles of turbulent kinetic energy and heat turbulent fluxes in the period of the plume passage by the tower.
\end{abstract}

Keywords: Atmospheric Boundary Layer; Dispersion; Turbulent Spectra; Atmospheric Turbulence. 


\section{Introdução}

Neste trabalho, são apresentados resultados do projeto "Sistema de monitoramento, análise e simulação da micrometeorologia e dispersão de contaminantes em uma região costeira”, em execução pela Universidade Federal de Santa Maria, com suporte financeiro da Telemétrica Viana SA (TEVISA) e da Linhares Geração SA (LGSA). Uma torre micrometeorológica de 140 m de altura, com observações em vários níveis verticais está em operação junto a área da Usina Termoelétrica da LGSA. A altura e quantidade de níveis de observação conferem a essa torre um ineditismo a nível internacional no que se refere à observação do ambiente em que ocorre a dispersão de poluentes emitidos por uma Usina Termoelétrica. Em particular, o ambiente escolhido apresenta características interessantes, pois a torre está a aproximadamente $3 \mathrm{~km}$ da costa oceânica, permitindo a observação detalhada da interação dos processos de camada limite marítima e terrestre no local, e de como estes afetam a dispersão dos escalares emitidos pela Usina. O caráter inovador e inédito da iniciativa permitirá compreender e descrever características ainda não foram conhecidas pela comunidade científica acerca dos ambientes de dispersão de escalares emitidos por fontes pontuais. $\mathrm{O}$ projeto apresenta, portanto, tanto um âmbito de pesquisa básica como de pesquisa aplicada.

A torre foi instalada ao norte da Usina, direção de onde provém o vento predominante, com o objetivo principal de caracterizar o ambiente em que a dispersão ocorre antes deste ser perturbado pela própria Usina. Porém, a grande proximidade entre a torre e a fonte dos poluentes, também permite que se observem eventos em que, sendo o vento predominante de Sul, a pluma de emissão passa diretamente pela torre. Estes eventos são analisados em maior detalhe neste trabalho, que com isso apresenta as primeiras observações diretas da turbulência no interior da pluma de emissão de uma Usina. Uma primeira pergunta a ser respondida é se os sinais observados são de fato turbulentos. A enorme relevância desta questão reside no fato de que, em caso positivo, como demonstrado neste trabalho, técnicas de análise de sinais turbulentos poderão ser aplicadas para se determinar grandezas como a energia cinética turbulenta e o fluxo de calor no interior da pluma. Este conhecimento permitirá, no futuro, estabelecer modelos conceituais de como a pluma modifica o próprio ambiente em que ela está se dispersando.

\section{Metodologia}

A torre micrometeorológica foi instalada em agosto de 2016, e vem operando continuamente desde então. Inicialmente, ela contava com 11 níveis de medida de turbulência, em 1, 2, 5, 9, 20, 37, 56, 75, 94, 113 e 132 m. Estas medidas são feitas por anemômetros sônicos tridimensionais, modelo CSAT3B da Campbell Sci. Inc, proporcionando observações das três componentes do vento e de temperatura com alta frequência, de $10 \mathrm{~Hz}$. O sônico de $56 \mathrm{~m}$ apresentou problemas a partir de duas semanas de operação, parando de funcionar em novembro de 2016, quando esse problema comprometeu todo o sistema, interrompendo as medidas por 3 semanas. A partir de dezembro de 2016 as medidas foram reiniciadas e o sônico de $1 \mathrm{~m}$ substituiu o de $56 \mathrm{~m}$, de forma que a partir de então são 10 os níveis de turbulência disponíveis. Também são feitas observações médias de temperatura e umidade específica do ar a cada minuto, em 10 níveis verticais: 2, 10, 20, 30, 50, 70, 90, 110, 130 e 140 m. Um perfilador de vapor d'água e dióxido de Carbono proporciona observações deas concentrações médias dessa grandezas a cada meia-hora nos níveis de 1, 2, 5, 10, 15 e $20 \mathrm{~m}$. Entretanto, essas medidas começaram apenas em dezembro de 2016, não sendo utilizadas no presente estudo.

O presente estudo foca em eventos nos quais o vento soprou da Usina para a torre, proporcionando eventos em que o ar proveniente da pluma de emissão fosse diretamente amostrado. Foi escolhido um período de 120 min noturno com mudanças abruptas na direção do vento para o quadrante Sul. Desta maneira, os intervalos de tempo em que as medidas são afetadas pela passagem da pluma, trazida pelo vento vindo da direção da Usina, podem ser nitidamente identificados. Essa abordagem possibilita uma visualização bem precisa dos efeitos da pluma na região da torre, já que as condições dessa região antes da chegada da pluma podem ser determinadas e adotadas como uma condição de normalidade ou de controle.

Com o objetivo de identificar as características da turbulência dentro da pluma e os efeitos causados por ela nas regiões mais próximas, parâmetros que quantificam ou caracterizam a turbulência, assim como o transporte turbulento, na camada limite planetária (CLP) serão estimados nos períodos de controle e de passagem da pluma pela torre para fins de comparação. Valores médios, desvios padrões $(\sigma)$, energia cinética turbulenta $(E C T)$, fluxo cinemático de calor sensível $\left(\overline{w^{\prime} T^{\prime}}\right)$, velocidade de fricção $\left(u_{*}\right)$ e parametro de estabilidade $(z / L)$ são calculados a partir das medidas de alta frequência realizadas na torre Stull (1988).

Um dos principais resultados dos trabalhos publicados por A. N. Kolmogorov em 1941 foi a lei de potência para o sub-intervalo inercial, ou lei dos $-5 / 3$ para o espectro de energia Frisch (1995). A verificação da lei de potência dos -5/3, ou -2/3 se o espectro for normalizado pela frequência, fornece uma maneira de identificarmos se os espectros calculados para as medidas na presença da pluma representam flutuações turbulentas. No presente trabalho, a relação de Kolmogorov será utilizada para identificar se as séries obtidas no interior da pluma de emissão da Usina são sinais turbulentos ou não. Essa é uma questão importante porque esta é a primeira vez que observações deste tipo são apresentadas. Como ao ar no interior da pluma é perturbado por um forte aquecimento, não se tem conhecimento de suas características, e se os métodos empregados para a análise de sinais em parcelas não perturbadas de ar podem ou não ser utilizados neste caso. 


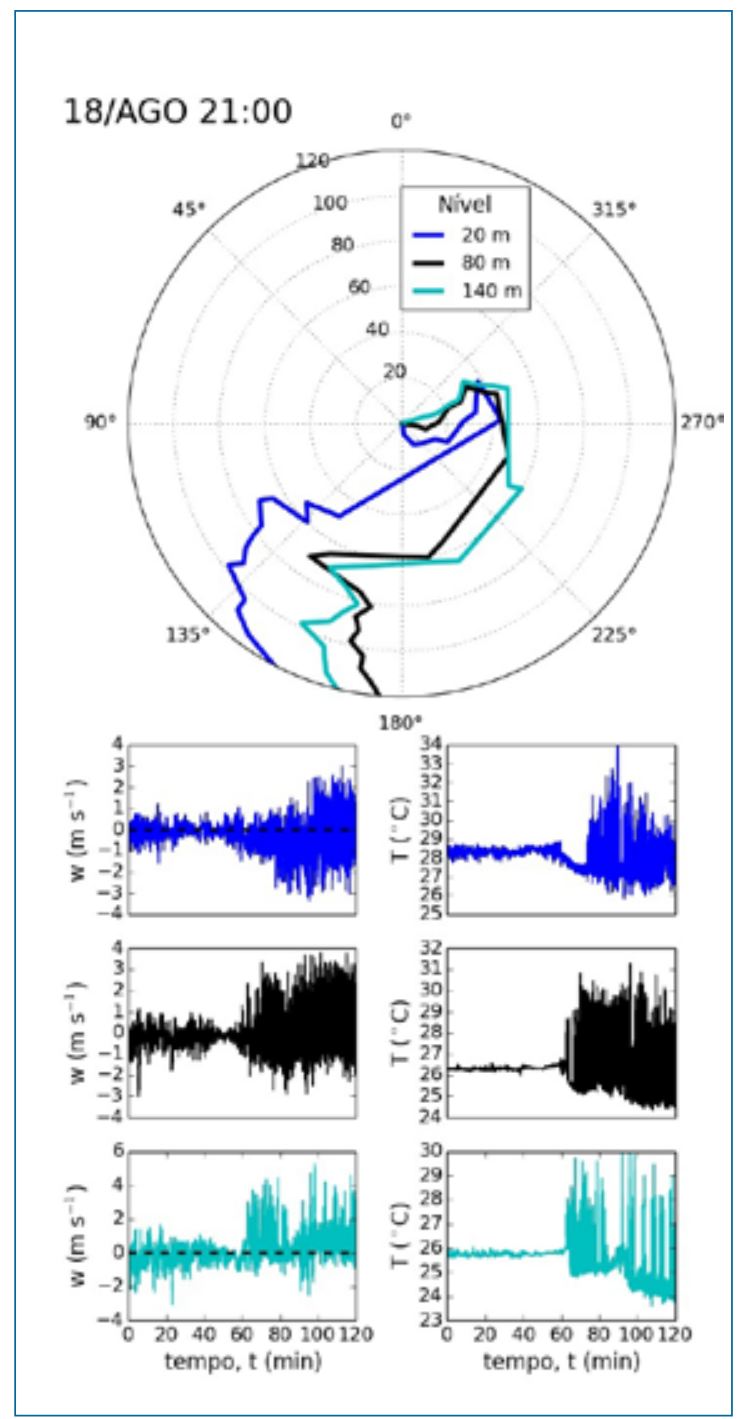

Figura 1: Direção do vento médio e medidas da componente vertical da velocidade do vento $(w)$ e temperatura $(T)$, realizadas nos níveis de 20, 75 e $140 \mathrm{~m}$ em um caso noturno, a partir das 21:00 do dia 18 de agosto de 2016.

\section{Resultados obtidos}

Para o período selecionado, calculou-se a direção horizontal média do vento para intervalos de 5 min. Na Figura 1 , pode-se observar uma considerável variação na direção do vento no intervalo de tempo compreendido entre 40 e 60 min, com os ventos deixando de se originarem da direção leste, e passando a virem do quadrante Sul durante o restante do período nos níveis de 75 e $140 \mathrm{~m}$, e da direção sudoeste em $20 \mathrm{~m}$. A presença da pluma nas proximidades da torre pode ser facilmente observada nas medidas da componente vertical do vento $(w)$ e da temperatura $(T)$ através do aumento abrupto nas flutuações dessas grandezas (Figura 1). Nos níveis mais altos apresentados (75 e $140 \mathrm{~m}$ ), a passagem da pluma quente é detectada pelos instrumentos aproximadamente no tempo de $60 \mathrm{~min}$. A $20 \mathrm{~m}$, onde o vento é de Sudoeste, o aumento das flutuações devido à presença da pluma quente é observado em aproximadamente $70 \mathrm{~min}$. Esse tempo de atraso observado em relação aos níveis superiores pode estar associado à transferência da energia trazida pela pluma para os níveis mais baixos. Podemos admitir que o aumento das flutuações nas variáveis turbulentas à $20 \mathrm{~m}$ não é causado por interação direta com a pluma, já que a variação abrupta de $130^{\circ}$ na direção do vento entre 40 e 60 min aparentemente não afetou as medidas de $w$ e $T$. Dentre os níveis apresentados na Figura 1, o mais afetado pela pluma foi o de $75 \mathrm{~m}$. Isto é esperado, pois o topo da chaminé encontra-se a $40 \mathrm{~m}$. No entanto, o mecanismo que transfere a energia introduzida pela massa de ar da pluma para o níveis mais baixos ainda deve ser melhor investigado.

Alguns parâmetros que caracterizam o escoamento na CLP foram utilizados para avaliar a alteração do escoamento nas proximidades da torre devido à presença da pluma. A Tabela 1 apresenta os valores obtidos para o período ao nível de $75 \mathrm{~m}$. Estes parâmetros foram estimados levando em consideração a estatística das medidas de dois intervalos distintos. O primeiro, sem presença da pluma, compreende os primeiros $60 \mathrm{~min}$ das series de medidas. O segundo intervalo é composto pelos 60 min restante, 


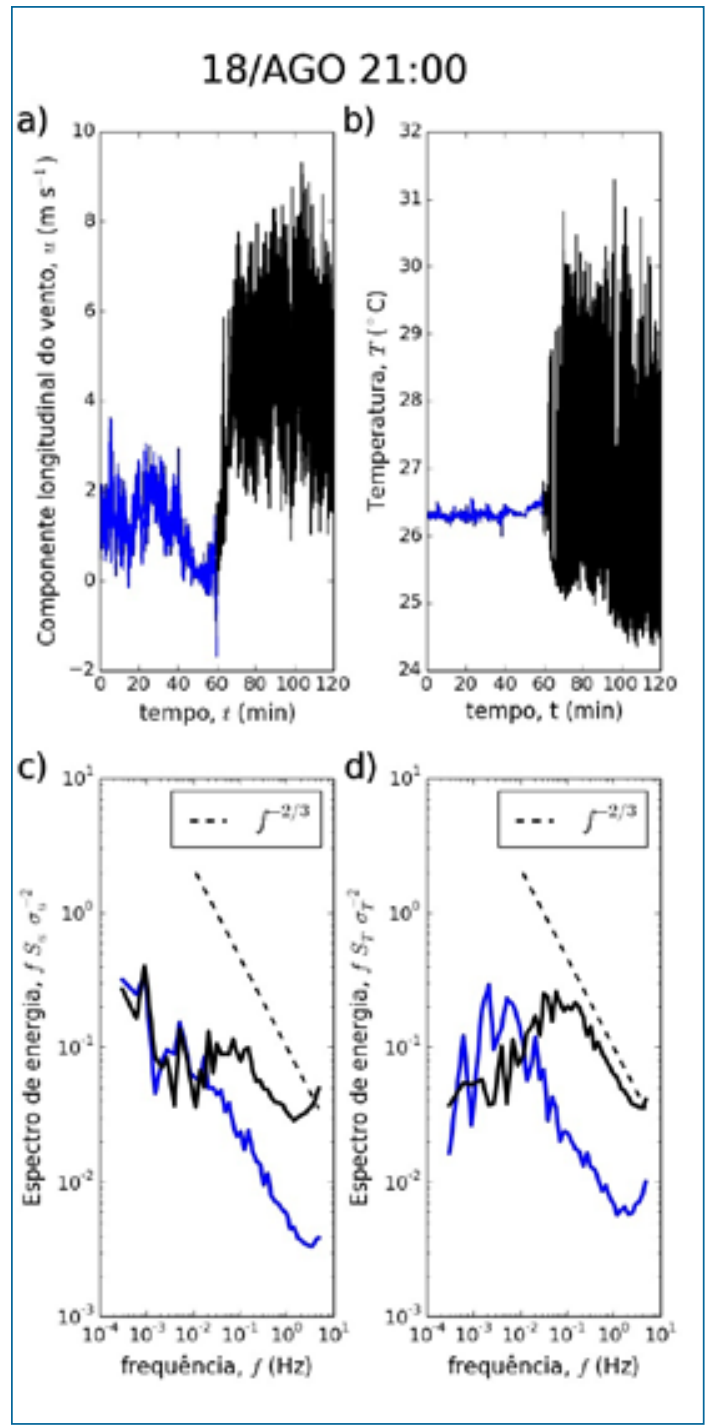

Figura 2: Medidas da componente longitudinal do vento (a) e de temperatura (b) $(75 \mathrm{~m})$ e os respectivos espectros de energia (c e d).

onde claramente podemos observar os efeitos da passagem da pluma.

Tabela 1: Parâmetros associados ao escoamento observado ao nível de $75 \mathrm{~m}$ calculados para o período (18/AGO 21:00). O segundo intervalo foi o caracterizado pela passagem da pluma.

\begin{tabular}{lccccccccc}
\hline & $\begin{array}{c}\text { Direção } \\
\text { (graus) }\end{array}$ & $\begin{array}{c}\bar{u} \\
(\mathrm{~m} / \mathrm{s})\end{array}$ & $\begin{array}{c}\sigma_{u} \\
(\mathrm{~m} / \mathrm{s})\end{array}$ & $\begin{array}{c}\sigma_{v} \\
(\mathrm{~m} / \mathrm{s})\end{array}$ & $\begin{array}{c}\sigma_{w} \\
(\mathrm{~m} / \mathrm{s})\end{array}$ & $\begin{array}{c}\sigma_{T} \\
\left({ }^{\circ} \mathrm{C}\right)\end{array}$ & $\begin{array}{c}\overline{w^{\prime} T^{\prime}} \\
\left(\mathrm{m}^{\circ} \mathrm{C} / \mathrm{s}\right)\end{array}$ & $\begin{array}{c}u_{*} \\
(\mathrm{~m} / \mathrm{s})\end{array}$ & $\mathrm{z} / \mathrm{L}$ \\
\hline Intervalo 1 & 271 & 1,13 & 0,72 & 0,69 & 0,36 & 0,07 & $-0,009$ & 0,18 & 17,21 \\
Intervalo 2 & 167 & 4,56 & 1,44 & 0,91 & 0,90 & 1,197 & 0,540 & 0,40 & $-97,72$ \\
\hline
\end{tabular}

A passagem da pluma causou um aumento considerável nos desvios padrões das componentes da velocidade do vento $\sigma_{u, v, w}$, e consequentemente na $E C T(182 \%)$, e na velocidade de fricção $u_{*}(222 \%)$. As mudanças mais expressivas foram observadas nas variâncias de temperatura $\sigma_{T}(1700 \%)$ e da componente vertical da velocidade do vento $\sigma_{w}(250 \%)$, resultando em um aumento também expressivo no módulo do fluxo cinemático de calor sensível $\overline{w^{\prime} T^{\prime}}(6000 \%)$, além da inversão no sentido do fluxo. Este, é normalmente negativo em condições noturnas, devido à transferência de calor da atmosfera para a superfície no período. Entretanto, no interior da pluma aquecida, fluxos positivos (para cima), e de grande magnitude ocorrem. O parâmetro de estabilidade estática $(z / L)$ Panofsky e Dutton (1983) calculado no nível de 75 m variou de aproximadamente 17 (camada estável) para -97 (camada instável). Ou seja, mesmo ocorrendo um aumento de, a turbulência observada durante a passagem da pluma é predominantemente gerada por forçante convectivo.

Os resultados apresentados até o momento indicam que a pluma não é um elemento passivo, mas que existe uma intensa 


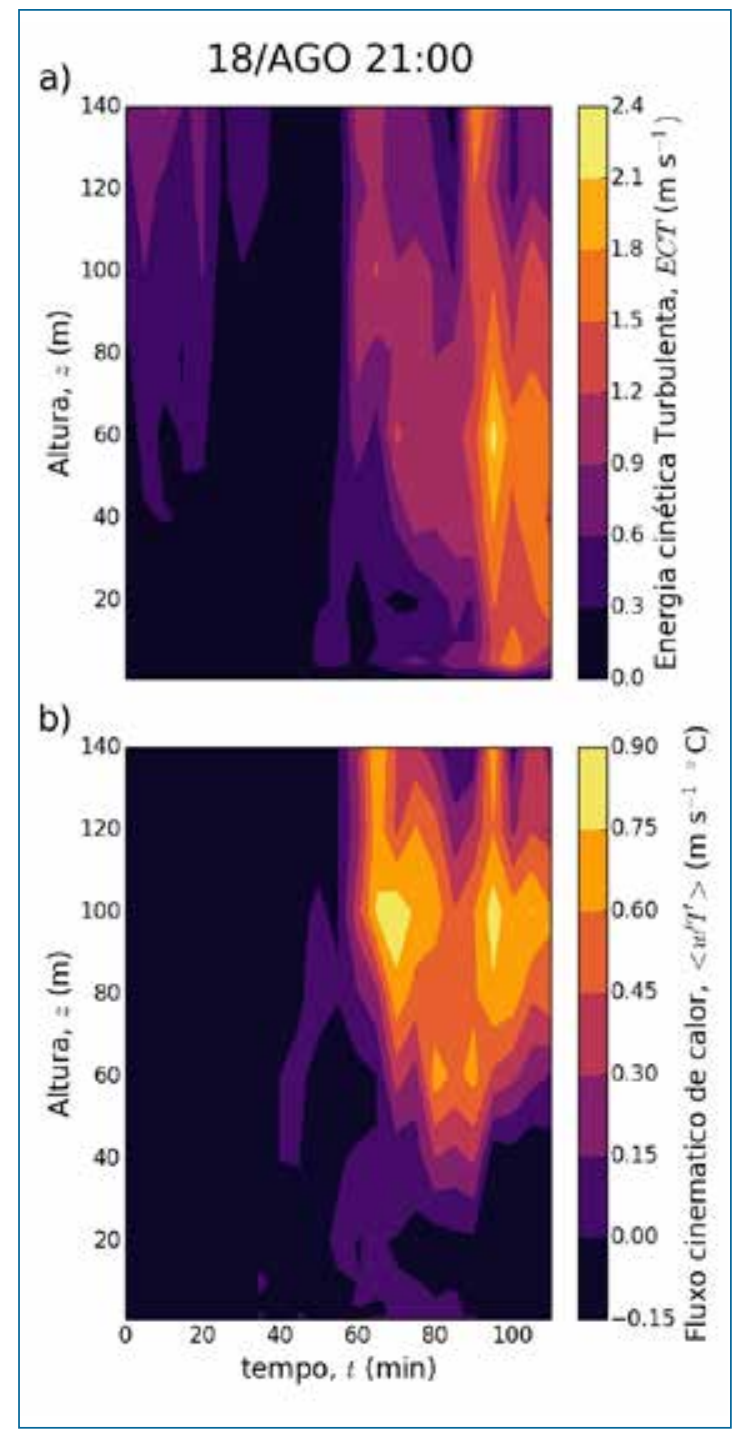

Figura 3: Evolução temporal dos perfis verticais da energia cinética turbulenta (a) e de fluxo cinemático de calor sensível (b) observados durante o período selecionado.

interação entre ela e a região que a cerca. No entanto, é importante certificar se as alterações observadas no escoamento são genuinamente consequência de eventos turbulentos ou se são estatísticas espúrias causadas pela passagem da pluma. Com este propósito, calculou-se o espectro de energia para intervalos com e sem interferência da pluma. Foram adotados os mesmos subintervalos utilizados na análise apresentada nas Tabela 1. Na Figura 2(a) e 2(b) apresentamos as medidas da componente longitudinal do vento e de temperatura obtidas ao nível de $75 \mathrm{~m}$. Os respectivos espectros de energia normalizados são apresentados nas Figuras 2(c) e 2(d). Em ambos espectros, da componente longitudinal da velocidade do vento $(u)$ e da temperatura $(T)$, é evidente que a passagem da pluma introduz uma considerável quantidade de energia nas altas frequências, que já era possível deduzir das séries temporais apresentadas na Figura 1. A pluma altera a distribuição de energia e, consequentemente, as escalas dos turbilhões mais energéticos. Porém, o resultado mais importante no contexto do presente trabalho é que, mesmo ocorrendo uma alteração completa no espectro de energia, se pode observar que a lei de potência para o subintervalo inercial $\left(f^{-2 / 3}\right)$ é mantida. Isso atesta que as grandes flutuações observadas na passagem da pluma são de origem turbulenta.

Uma das principais contribuições do presente projeto foi a possibilidade de realização de medidas de qualidade em diferentes alturas próximas à usina. Experimentos dessa magnitude são raramente executados e são fundamentais para a compreensão dos escoamentos na CLP. Além disso, os dados obtidos possibilitam que a dinâmica da pluma oriunda da usina seja analisada sobre uma nova ótica.

A partir da comprovação do caráter turbulento do sinal observado, indicada nas Figuras 2, é possível determinar diferentes grandezas associadas ao estudo de séries turbulentas, e como elas são perturbadas pela presença da pluma de emissão da Usina Como exemplos, são apresentados os perfis verticais de $E C T$ e do fluxo de calor para o período (Figura 3). O início da passagem da pluma em 60 min causou um aumento mais intenso nas regiões mais altas dos perfis, tanto na ECT (Figura 3(a)) quanto em 
$\overline{w^{\prime} T^{\prime}}$ (Figura 3(b)). No entanto, um máximo de fluxo foi encontrado pouco tempo após a chegada da pluma localizado entre os níveis de 75 e $100 \mathrm{~m}$, enquanto que para a $E C T$, o máximo observado nesse intervalo ocorreu após os 90 min em um nível mais baixo $(60 \mathrm{~m})$. Nos últimos 30 minutos, o perfil de $\overline{w^{\prime} T^{\prime}}$ fica dividido aos $40 \mathrm{~m}$, com fluxo positivo, característico da presença da pluma, acima desse nível e fluxo negativo, característico do estado médio da camada limite noturna, abaixo.

\section{Discussões e perspectivas}

A torre micrometeorológica de Linhares representa um marco científico no que se refere à observação do ambiente em que ocorre a dispersão de poluentes de uma Usina Termoelétrica. A instrumentação ali instalada e a grande altura dessa torre lhe conferem um potencial semelhante ao dos mais importantes experimentos micrometeorológicos do planeta. Pela primeira vez, uma capacidade de observação deste nível foi instalada junto a uma Usina Termoelétrica. Desta forma, é seguro afirmar que os resultados do projeto aqui descrito tem potencial para proporcionar uma grande melhora na compreensão de processos difusivos. No presente trabalho, se analisou um aspecto em particular, que é as observações das plumas de poluentes diretamente sobre a torre. Não se tem notícia de observações semelhantes na literatura, de forma que este estudo é inédito a nível internacional. Os resultados mostraram que enormes flutuações das componentes do vento e da temperatura ocorrem quando o vento sopra diretamente da Usina para a torre. Mais importante ainda, apesar de ter características próprias e totalmente distintas das observadas em condições sem a presença da pluma, as séries temporais desta quantidade preservam as características de um sinal turbulento. Em particular, se observou de maneira evidente que os espectros de Fourier dos sinais seguem a Lei de Kolmogorov, apresentando uma lei de escala com a densidade espectral decaindo com o número de onda através de um expoente de $-5 / 3$.

Os resultados deste estudo são bastante preliminares. Apenas um caso particular foi considerado, com o propósito de comprovar o caráter turbulento do sinal observado durante a passagem da pluma e identificar qualitativamente aspectos associados à dispersão desta pluma em situações distintas. Estes objetivos foram alcançados. No prosseguimento deste trabalho, é necessário sistematizar essa análise, de forma a incluir uma quantidade maior de eventos. Nesse caso, a análise espectral será feita para garantir o caráter turbulento de cada série encontrada. Em um momento seguinte, é importante quantificar as características da dispersão da pluma, de forma a determinar as condições ambientes que afetam esta dispersão. Isso está planejado para as fases posteriores deste trabalho.

Uma vez que os eventos de observação da pluma sejam sistematizados, estes resultados terão uma grande aplicação para a simulação da dispersão de escalares no ambiente local. As observações feitas em períodos de vento de Norte, quando o ar passa primeiro da torre antes de chegar na Usina proporcionam uma caracterização extremamente detalhada da camada limite atmosférica não perturbada pela fonte. Por outro lado, as observações dos casos com vento de Sul, durante os eventos indicam as características da pluma e de como ela se mistura ao ambiente não perturbado. Essas duas informações podem alimentar modelos numéricos de simulação de escoamentos atmosféricos com fontes de escalares. Tais modelos permitirão compreender não apenas as características da dispersão a partir da Usina em questão, mas, principalmente, oferecer novas percepções sobre a física dos processos difusivos.

\section{Conclusões}

O estudo aqui apresentado é uma iniciativa pioneira em uma área do conhecimento de grande relevância científica na atualidade. A questão de dispersão de escalares tem enorme relevância ambiental e, ao mesmo tempo, por envolver processos afetados pelo fenômeno da turbulência, apresenta grandes incertezas que surgem de uma descrição incompleta do processo pelos modelos conceituais existentes. De maneira geral, os maiores avanços científicos ocorrem quando grandes experimentos observacionais são executados com o objetivo de analisar os problemas existentes em detalhe. É isso que está sendo proposto e feito no projeto presentemente descrito. Ainda que os resultados aqui mostrados sejam preliminares, em decorrência do estágio inicial do projeto, eles também são extremamente promissores. Aqui se mostrou que as observações diretas das características da pluma, inéditas na comunidade científica tem potencial para utilização futura, e que as características observadas estão qualitativamente em acordo com o conhecimento de física da camada limite planetária. No desenvolvimento do presente projeto, quando às observações se unirem desenvolvimentos teóricos e simulações numéricas com modelos matemáticos de escoamentos atmosféricos, este potencial será utilizado de maneira plena, e as características aqui apresentadas de maneira descritiva serão quantificadas e detalhadas.

\section{Referências}

Frisch, U. (1995). Turbulence: the legacy of AN Kolmogorov. Cambridge university press.

Panofsky, H. A., Dutton, J. A. (1983). Atmospheric turbulence. John Wiley.

Stull, R. B. (1988). An introduction to boundary laver meteorology, vol 13. Springer Science \& Business Media, Dordrecht. 
Luís Gustavo Nogueira Martins

Otávio Costa Acevedo

Franciano Scremin Puhales

Pablo Eli Soares De Oliveira

Cláudio Alberto Teichrieb

Samuel Silva

Giuliano Demarco
Universidade Federal de Santa Maria, Santa Maria, RS, Brasil

E-mail: lgnm.sm@gmail.com

Universidade Federal de Santa Maria, Santa Maria, RS, Brasil E-mail: otavio@ufsm.br

Universidade Federal de Santa Maria, Santa Maria, RS, Brasil E-mail: fpuhales@gmail.com

Universidade Federal de Santa Maria, Santa Maria, RS, Brasil E-mail: pablo.deoliveira@gmail.com

Universidade Federal de Santa Maria, Santa Maria, RS, Brasil E-mail: teichrieb@gmail.com

Universidade Federal de Santa Maria, Santa Maria, RS, Brasil E-mail: samueltec@gmail.com

Universidade Federal de Santa Maria, Santa Maria, RS, Brasil E-mail: giulianofisico@gmail.com

Felipe Denardin Costa
Universidade Federal de Santa Maria, Santa Maria, RS, Brasil E-mail: fdenardin@gmail.com 